\title{
Design of an Extensive Emergency Rescue Service for Electric Vehicles
}

\author{
Junghoon Lee, Hyobin Kim, and Gyung-Leen Park* \\ Dept. of Computer Science and Statistics, \\ Jeju National University \\ 690-756, Jejudaehakno 102, Jeju City, Jeju-Do \\ Republic of Korea \\ \{jhlee,glpark\}@jejunu.ac.kr,hyobin914@infomind.co.kr
}

\begin{abstract}
This paper designs and develops an emergency rescue service for electric vehicles (EVs), which may suffer from the battery depletion during their trips. After building relevant data models on both chargers and EVs, our design defines the interaction among three parties of EV drivers, the rescue server, and rescue vehicles to responsively process the dispatch request and systematically meet the user requirement. Particularly, a comprehensive mobile application is developed on the Android operating system to help users to promptly send a rescue request to the server and monitor the request processing status. This user interface allows EV drivers to automatically specify their locations and necessary personal information as well as to view the distribution of emergency vehicles through user-friendly maps. Our design is scalable for a large number of emergency vehicles and rescue requests, accelerating the wide deployment of EVs in our daily lives. Moreover, the rescue system design can collaborate with an EV tracking system and charge reservation agents to create a more sophisticated EV information service.
\end{abstract}

Keywords: electric vehicle, emergency rescue service, helper application, dispatch processing

\section{Introduction}

Intelligent computer algorithms make the power system smart, bringing the age of smart grid [1]. They achieve various optimization goals in the chain of power generation, transmission, distribution, and finally consumption [2]. As the electricity must be usually consumed as soon as it is generated, an optimal generation plan is indispensable for energy efficiency and system safety. Meanwhile, with the gradual penetration of EVs (Electric Vehicles), which are powered by electricity supplied from the grid and stored in their batteries, transportation becomes a part of the power network [3]. Due to their unique consumption behavior induced by the energy storage device, the role of two-way interaction between EVs and the grid gets much more important. It will be the bridge through which a sophisticated control strategy can be conveyed to EVs in response to monitored status reports from EVs, providing a run-time platform for diverse distributed applications.

\footnotetext{
* Prof. Gyung-Leen Park is the corresponding author.

This research was supported by the 2014 scientific promotion program funded by Jeju National University.
} 
In the meantime, the large deployment of EVs cannot be expected without an intelligent information service especially supporting systematic maintenance and tour planning [4]. It can be built upon comprehensive interoperability between diverse grid entities such as EVs, charging facilities, tracking systems, power networks, and so on [5]. Here, it must be mentioned that the highest priority is put on EV charging to overcome the short driving range and long charging time. In spite of the last improvement in battery manufacturing technologies, a fully-charged vehicle can drive just $100 \mathrm{~km}$ in practice. Many countries are making an effort to build such an information infrastructure for EVs, while the Internet of Energy (IoE) for Electric Mobility is an example of the information framework developed by a European research project [6]. According to their experience, EV services are required to include mobile applications to deal with the inherent mobility of EVs, taking advantage of a variety of abundant information collected from each smart grid entity.

Nowadays, mobile phones have remarkable computing performance, potentially interfacing with diverse on-board devices [7]. Moreover, it is not uncommon for mobile phones to be equipped with more than on CPU, enhancing its computer power via parallel processing. They can work as not only an in-vehicle computer but also a gateway device seamlessly communicating with remote high-end servers via ubiquitous wireless channels. Wireless channels currently available to moving EVs are cellular and WiFi networks. After all, it is possible to build a large scale information service, in which high-performance computers carries out complex data processing procedures dealing with a variety of information [8]. On the other hand, mobile applications interact with this server via welldefined communication protocols over the appropriate wireless channel. Particularly, their display units provide rich graphic interfaces to drivers through an appropriate mobile application developed by Android and iOS programming. These applications, running inside EVs, are essentially location-based and thus benefit from map utilities [9].

In this paper, we design and develop a mobile helper application for EVs, particularly focusing on the emergency rescue and the driving activity collection. EVs discharged enroute, possibly due to unexpected power consumption and unavailability of charging facilities, want electricity to be supplied as prompt as possible. Such a service can alleviate the range anxiety problem, hosting diverse EV information services [10]. The mobile application can be downloaded anytime to the driver's phone or the in-vehicle computer device. This service must be built upon an efficient data processing framework embracing EV tracking systems, charger status monitoring mechanisms, and other real-time data handlers. As an extended version of our previous work [11], this paper includes more details on the system configuration regarding database design, request processing mechanisms and cooperation with other services, not just confined to the EV helper applications running on a mobile terminal.

This paper is organized as follows: After issuing the problem in Section 1, Section 2 introduces related work for EV tracking, monitoring, and rescue systems. Section 3 shows the important database definitions for our emergency rescue system. Section 4 explains the operation of the designed rescue system and the interaction mechanism between the back-end server and mobile applications. It also demonstrates the user interface implemented on Android phones. Finally, Section 5 summarizes and concludes this paper with a brief introduction of future work.

\section{Related Work}

Targeting at metropolitan areas, such as London, [12] develops first an elaborate simulator capable of evaluating emergency rescue dispatch algorithms, mainly for ambulances. The authors build the Combined Automatic Dispatch Model (CARD) to delegate rescue units to 
incidents and standby points. Essential requirements for positioning rescue vehicles are identified and combined with each other, completing a set of five weighted basis functions. Those weighted functions enumerate the number of incidents that will occur at any one location, incident density, and unit coverage at grid locations. Here, two grids are created. The first grid contains a static pre-computed map of incident density, while the second grid contains the number of rescue units that can reach within a specific time bound. Then, the cumulative sum is normalized to identify those areas having a high incident density. This scheme is a good example for how to distribute EV rescue vehicles over the target area. Moreover, it is emphasized that emergency response units must be equipped with ubiquitous computing technologies for sensing, resilient communication, and command-and-control infrastructure for cooperating with transport systems.

As for communication-based EV fleet management, [13] deals with the integration of a model-based fault diagnosis and corresponding fault control of an intelligent heavy-size autonomous vehicles. Here, the available information for fault diagnosis includes personal localization of the vehicle, kinematic and dynamic state of the surrounding environment, and station of the traction and steering control. Moreover, each vehicle can communicate in V2V (Vehicle-to-vehicle) or V2I (Vehicle-to-Infrastructure) types, possibly accessing the coordinates of the trajectory. Basically, its model-based fault detection compares the real behavior of a physical system with the values calculated from a mathematical model. The diagnosis procedure generates analytic redundancy relations. They form a fault signature matrix, which represents the influence of the discrepancy between the real behavior and the mathematical estimation. Finally, the fault-tolerant control can be accomplished via either system reconfiguration or fault accommodation.

From the viewpoint that a CPS (Cyber-Physical System) is characterized by a tight interaction between computing devices and the physical environment, [14] addresses that EVs introduce new CPS components like batteries, electric motors, and energy management. EV platforms are expected to shift towards a fully time-triggered system for deterministic operation, especially for safety ensuring. To this end, the EV control system requires novel bus architecture, computing devices, and operating systems. The authors propose a multilayered task scheduling scheme which copes with the complexity of the EV physical system by separating the optimization in different steps. In their design, the problem of resource allocation and configuration, task mapping, and message routing are associated with a set of linear constraints with binary variables. In addition, the new component-specific interaction mechanisms are designed for distributed battery management and distributed control verification.

As an example of EV tracking application, during the process of developing a modeling and controlling framework for EV energy management, [15] builds a vehicle tracking system, coping with uncertainties associated with power generation and EV population. The two-way communication, based on the tracking capability, is essential for the potential EV control as a dispatchable energy source. The electricity charged to and stored in EV during the low-load hours is sent back to the grid, reducing stress during peak hours and integrate more renewable energy. Specifically, the authors develop not only a sliding mode control strategy in nonlinear control literature but also Monte Carlo simulations. The demand-side load control forces the aggregate power demand to track a desired power trajectory. For more sophisticated control, the SoC (State of Charge) for each vehicle can be monitored via the tracking system. This mechanism can proactively prevent an EV from being fully discharged by notifying an alarm to the vehicle.

Besides, AAA (American Automobile Association) deploys mobile charging units dedicated to EVs to its roadside assistance operations and is now expanding its service to 
more cities. Each unit is well-equipped with fast chargers capable of powering up an EV in a few minutes. For example, the mobile charging trucks create charging power through an onboard generator that is powered also by gasoline or alternative fuels. As the rescue vehicle usually has enough time, the mobile charger can fill its battery even from the intermittent renewable energies. It can handle the needs of most EVs as it supplies electricity for an energy-depleted EV to reach a nearby charging station. This service will provide 10 to 15 minutes of charge time to members, enough to get them to a charging station within 3 to 15 miles. Here again, the specific control strategy can take advantage of cyber-physical system approaches in processing a variety of status monitoring data and taking an appropriate action [16]. Here, respective battery operations can be considered control activation.

\section{Database Design}

Figure 1 describes our data model of a relational database. It depicts the 4 important tables, namely, charging station status, charger status, dispatch request transaction, and rescue request transaction.

\begin{tabular}{l}
\multicolumn{1}{c}{ Charging station } \\
\hline ID \\
name \\
address \\
phone number \\
number of chargers in operation \\
number of EVs waiting in queue \\
latitude \\
longitude
\end{tabular}

Dispatch action

\section{dispatch ID}

report sequence

emergency vehicle ID

availability

estimated arrival time

operation result

additional comment on dispatch

registration ID

registration date

\begin{tabular}{ll}
\multicolumn{1}{c}{ Charger } \\
\hline ID \\
name \\
station ID \\
charger model \\
charging type (AC, DC) \\
current status \\
latitude \\
longitude
\end{tabular}

Rescue request

request ID
request type
requester's phone number
additional comment on report
$\mathrm{EV}$ plate number
$\mathrm{EV}$ model
longitude
latitude
reservation time
rescue type
registration date

Figure 1 Service Scenario

Actually, our data model definition consists of a variety of tables such as user registration, vehicle model, necessary vehicle parts, management company, emergency vehicle, and the like. However, the listed 4 tables are most actively accessed by our rescue system. Here, a charger belongs to a charging station and this information is specified in the station ID field in the Charger table. Here, each table entry has both 
static and dynamic attributes. All of 4 tables have longitude and latitude fields to specify the location of an object. Currently, this location is neither bound to a road segment nor to a POI. As the table is maintained in the widely-used Oracle database, we can easily create spatial queries for more complex location-based applications.

First, the Charging station table includes not only static attributes such as ID, name, location, and the number of chargers, but also dynamic attributes such as the number of EVs waiting in its queue. Those dynamic fields are updated by the real-time monitoring system and synchronized in periodic basis. Here, a charger just connected to an EV is not in the active state. Next, the Charger table includes the records for individual chargers. Its main dynamic field is the current status which indicates whether the charger is currently injecting electricity to an EV. Next, a Rescue request table entry is created when an EV reports an emergency situation and requests to dispatch a rescue vehicle. For a request, the system fills the remaining fields after assigning a rescue vehicle. Finally, a Dispatch activity table entry is created each time a dispatch action is determined. On the completion of each dispatch operation, the results are recorded for a later analysis. Those two tables store the log data for emergency rescue requests and how they are processed.

\section{Emergency Rescue System}

\subsection{Emergency rescue scenario}

Our emergency rescue system builds a dedicated server, loosely coupled with other EV services. This server mainly retrieves relevant information from other applications like a tracking system when necessary. A rescue transaction is initiated by a rescue request from an $\mathrm{EV}$ and completed by the report from the rescue vehicle irrespective of the rescue operation type and result. Figure 2 depicts the overall service scenario of our emergency rescue system [11]. How it works can be explained by the flow of messages among three actors, namely, the EV which wants to request a rescue service, the emergency rescue server which coordinates the rescue transactions, and the emergency vehicle which will be dispatched to the requested spots. Here, the communication protocols between them are TCP/IP and SMS (Short Message Service). In addition, we assume that the necessary mobile application has been already downloaded to the cell phone of the EV driver before his or her trip.

1. An EV driver first sends a reservation request message to the emergency rescue server via the cellular network, specifically, CDMA (Code Division Multiple Access) in the Republic of Korea.

2. The rescue server acknowledges the receipt of the request as soon as it accepts the invocation.

3. The server checks the availability of rescue vehicles and selects the best one according to the distance to the requesting EV and the SoC (Status of Charge).

4. The picked rescue vehicle either accepts the order or reject. If rejected, the server repeats Step 3.

5. The server sends the reservation confirmation to the EV driver, who will now wait for the emergency vehicle, possibly doing other work. Here, the estimated arrival time will be informed also.

6. The rescue vehicle is dispatched and supplies the electricity enough for the EV to reach a nearby charging station. This time interval must be as short as possible for the rescue vehicle to move to another $\mathrm{EV}$. 
7. The rescue vehicle reports the completion of its service to the rescue server. Here, if the rescue vehicle cannot solve the problem, it may request a towing vehicle. The rescue server finalizes a transaction.

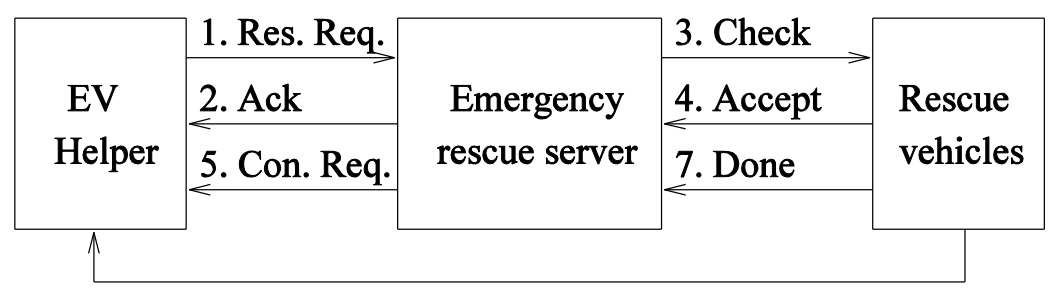

6. Dispatch an emergency vehicle

Figure 2 Service Scenario

Next, Figure 3 shows our mobile application implemented on the Android operating system. To begin with, Figure 3(a) shows the login page which also allows non-member login. However, with the member login, pre-registered information eases the rescue request process significantly. In addition, as shown in Figure 3(b), the initial menu allows the user to file an emergency rescue request, cancel the reservation, and connect the call center. For the emergency rescue request, most information fields including the caller name and the vehicle number, are preregistered while the current location of the vehicle can be automatically obtained from the embedded GPS receiver as depicted in Figure 3(c). Finally, Figure 3(d) shows the map-based display which marks the current locations of both the issuer vehicle and the rescue vehicle on the way. The respective coordinates are provided by the emergency rescue server which keeps tracking the current location of each rescue vehicle for an efficient dispatch. 


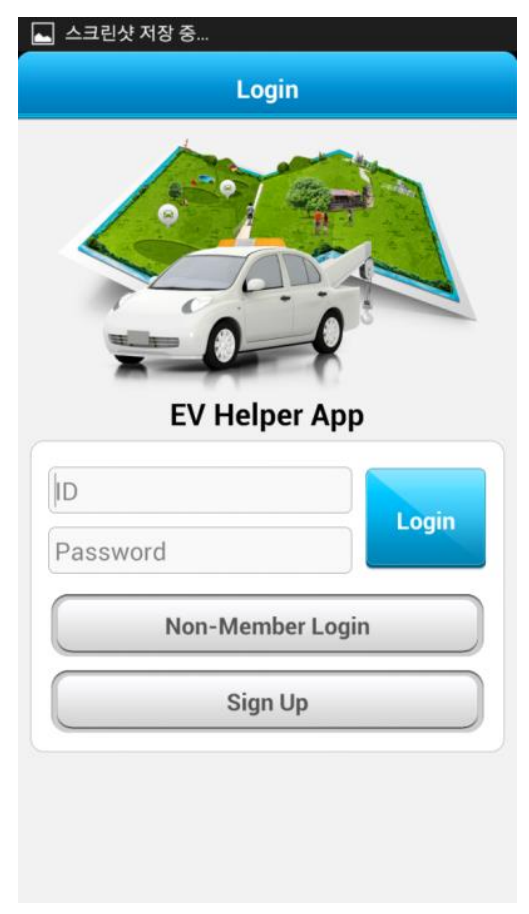

(a)Login

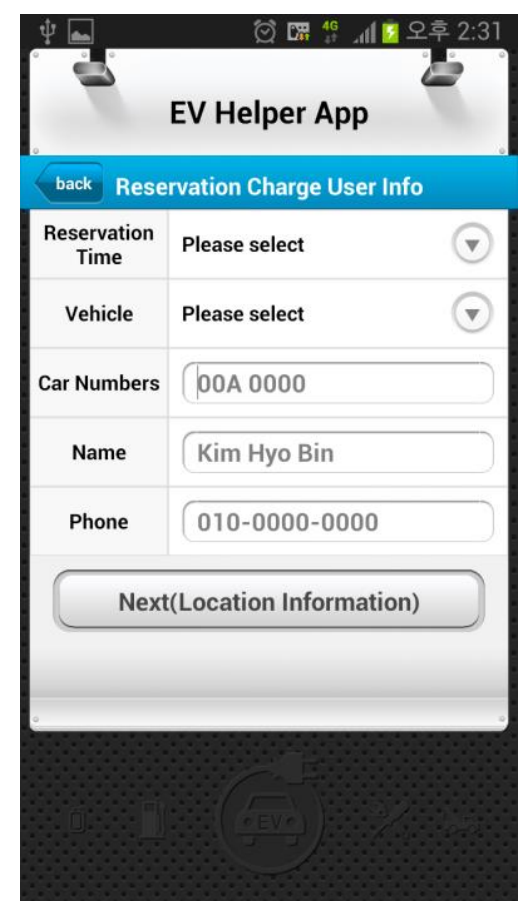

(c) Reservation request

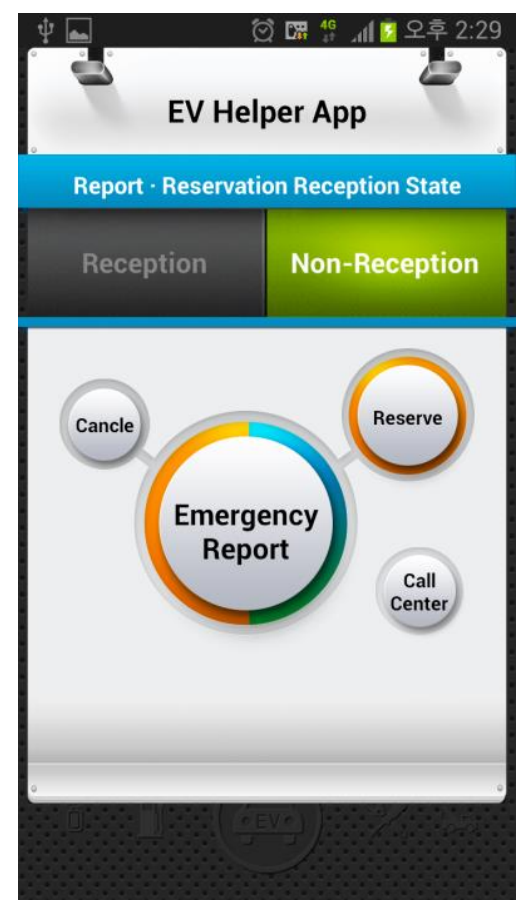

(b) Initial menu
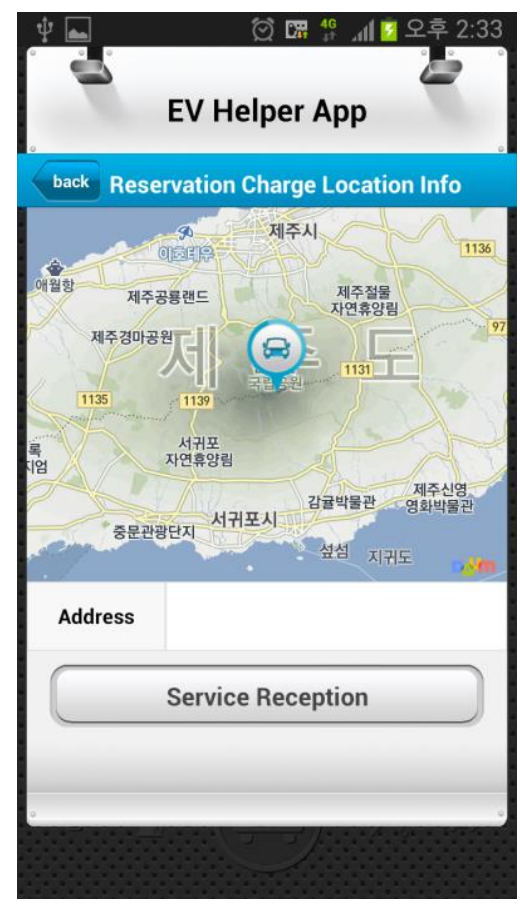

(d) Map interface

Figure 3 EV Helper Implementation 


\subsection{Control display}

Figure 4 shows the control display of the emergency rescue server. It tells the current locations of rescue vehicles and their operation status on the map of the target city area. When the operator selects an icon image on the map, the detailed information on the rescue vehicle pops up. It is essentially retrieved from the database table explained in Figure 1. The period of location reports from the rescue vehicle is a tunable parameter, which can be decided according to the communication cost and the accuracy level, and it is currently set to 1 minute. In addition, the rescue requester vehicles are also marked on this map. When the image is selected, the information is retrieved from the Rescue request table and will be displayed as shown in the right-hand side pop-up windows. The monitoring display helps the human manager grab the overall system operation and current dispatch activities. As the number of rescue vehicles and that of EVs on the city increase, it is necessary to employ an efficient spatial database.

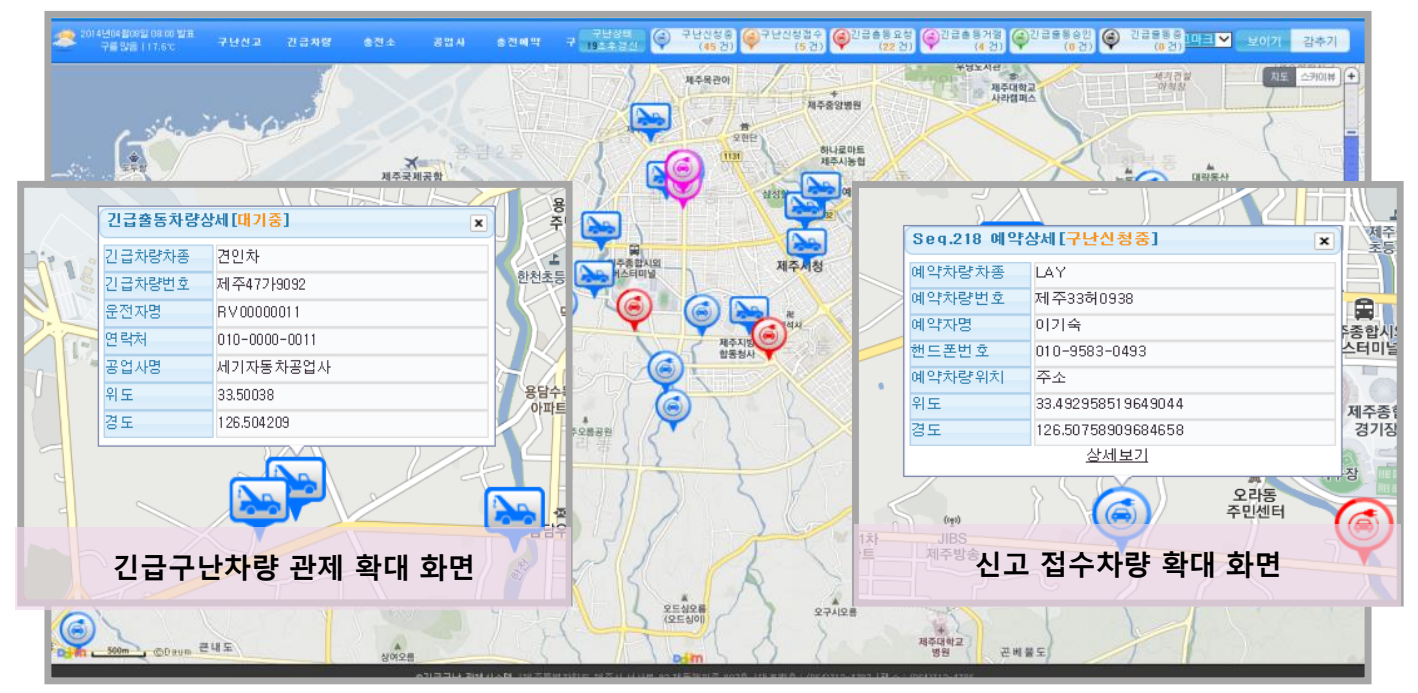

Figure 4 Server-Side Control Interface

Next, Figure 5 shows the Google map-based display on which the dispatch routes are plotted. For better efficiency, the dispatch order of a rescue vehicle to a set of rescue spots is very important to say nothing of how to assign a rescue vehicle to a rescue spot, especially when there are multiple requests pending simultaneously. The sequence decision belongs to a TSP (Traveling Salesman Problem) category in that it is advantageous to consider the total dispatch distance and the waiting time in the requester's aspect. The route that has been and will be taken by a specific rescue vehicle is plotted in this display to help to evaluate and improve a dispatch policy. To mark a route, we integrate road network comprised of nodes and links, which correspond to intersections and road segments, respectively. 


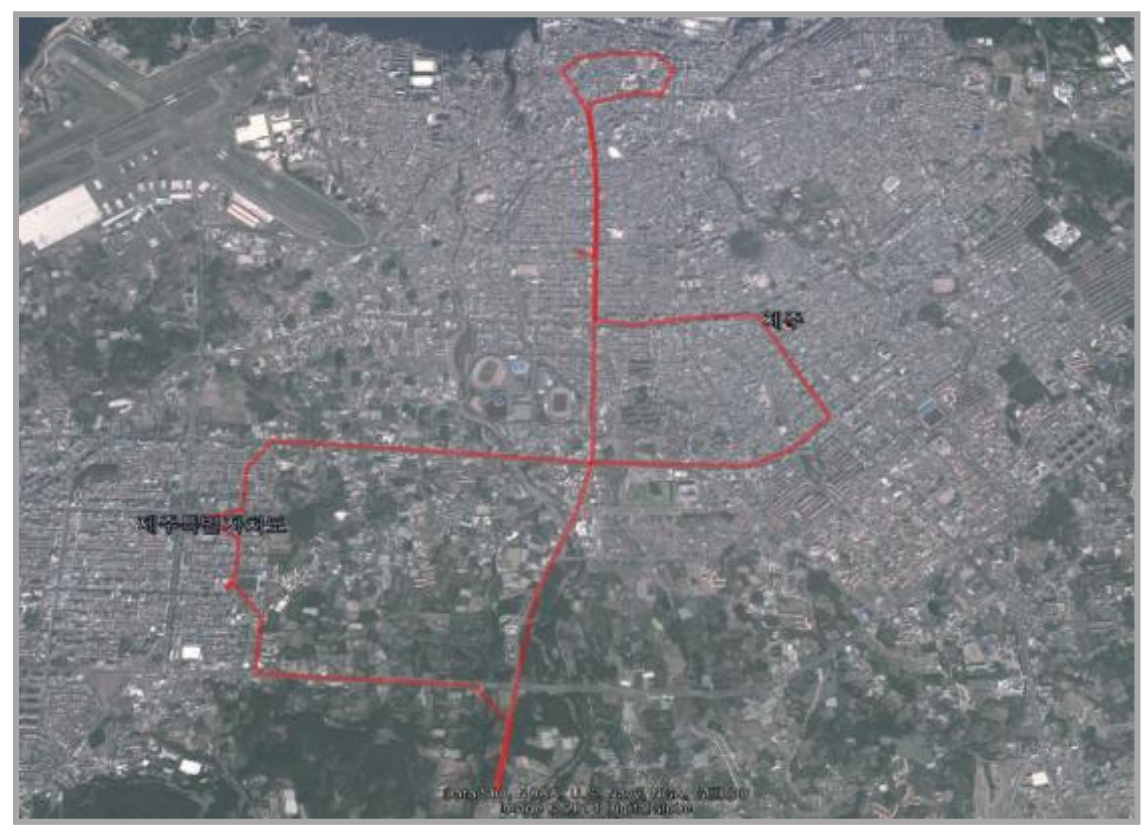

Figure 5 Map-Based Visualization

\subsection{Interaction with other services}

Figure 6 shows how the rescue service collaborates with an EV tracking system. To begin with, the tracking system keeps receiving location reports from respective EVs and updating their current positions. Nowadays, GPS signals are available almost every outdoor place, making it possible to build diverse location-based applications [17]. If necessary, the tracking system finds the road segment on which the EV is driving. This map matching is the prerequisite for efficient path finding and arrival time estimation. The location report also carries EV sensor readings which can be forwarded to the rescue system. Unlike the explicit request for the emergency report application, the status record is analyzed to detect an abnormal situation. Then, emergency rescue operation is automatically initiated. In this rescue path, the driver is just notified of what is going on and endorses the rescue operation. As for the status analysis, our system is accumulating emergency rescue $\log$ data to build comprehensive knowledge-base.

Next, the rescue vehicle supplies electricity to a discharged EV. To calculate the amount to supply, the rescue system must be aware of which station is closest to the rescue point. This information can be retrieved from a reservation agent of charging stations. The reservation agent may just check the current availability of chargers in the station [18]. In some cases, stations can run a charging operation scheduler which meets the constraint specified by charging requests, such as the amount to charge, deadline, actuation time, and the like [19]. Then, the charging operation can be regarded as a real-time task having its own execution time and deadline, except that multiple tasks can simultaneously run as long as the total consumption does not exceed the provisioned power [20]. After all, the emergency rescue system can enrich EV services by cooperating with other sophisticated information utilities. 
Hence, it is necessary for each service component to have a well-defined interface, which will create a new integrated service.

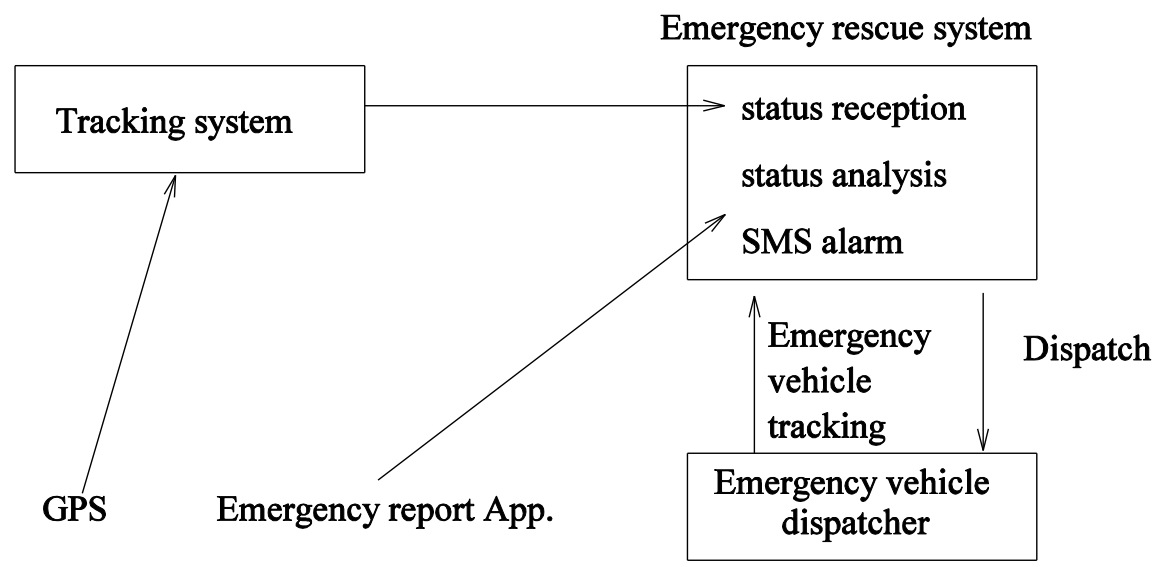

Figure 6 Interacting with a Tracking System

\section{Conclusions}

In the modern power system called the smart grid, in which EVs are one of the most vital elements, the information technology is essential for their intelligent management and operation. The EV helper application, implemented in this paper, makes it possible for EV drivers to overcome emergency situations mainly stemmed from battery depletion. The application provides a comprehensive user interface on mobile devices and the system design has developed a well-defined computing architecture in the emergency rescue system. The server-side control display exhibits the current rescue operation status, such as dispatch routes, via the map-based application. Our implementation can accommodate not only more invehicle sensors for more accurate diagnosis but also more emergency vehicles to be better responsive to emergency rescue requests. To this end, a variety of information can be efficiently managed and a sophisticated dispatch algorithm needs to be developed in the rescue server.

As future work, we are planning to design an efficient interaction mechanism with other services. The collaboration with an EV tracking system has been already exhibited and we believe that it is important to cooperate with charging agents. This is because the discharged vehicle needs to be charged at one of charging stations. Without a well-defined cooperation with reservation agents, the battery-depleted EV may wait too much time in a station having a long waiting queue. Moreover, a variety of EV services, for example, even in the area of V2G (Vehicle-to-grid), will appear and their integration can accelerate the penetration of EVs [21]. Particularly, V2G-enabled vehicles can sell their electricity on their trips and some of them can act as an emergency vehicle.

\section{Acknowledgment}

This paper is a revised and expanded version of a paper entitled "Development of an electric vehicle helper application for emergency rescue" presented at International Conference on Information Technology and Computer Science, Saipan, U.S.A., July 2014. 


\section{References}

[1] R. Prior, D. Lucani, Y. Phulpin, M. Nistor and J. Barros, "Network coding protocols for smart grid communications", IEEE Transactions on Smart Grid, vol. 5, no. 3, (2014), pp. 1523-1531.

[2] S. Ramchrun, R. Vytelingum, A. Rogers and N. Jennings, "Putting the 'smarts' into the smart grid: A grand challenge for artificial intelligence", Communications of The ACM, vol. 55, no. 4, (2012), pp. 86-97.

[3] D. Nguyen, L. Le, "Joint optimization of electric vehicle and home energy scheduling considering user comfort preference", IEEE Transactions on Smart Grid, vol. 5, no. 1, (2014), pp. 188-199.

[4] Y. Kobayashi, N. Kiyama, H. Aoshima and M. Kashiyama, "A route search method for electric vehicles in consideration of range and locations of charging stations", IEEE Intelligent Vehicles Symposium, (2011), pp. 920-925.

[5] Y. Hermans, B. Le Cun and A. Bui, "Individual decisions and schedule planner in a vehicle-to-grid context", International Electric Vehicle Conference, (2012).

[6] L. Bedogni, M. Di Felice, A. D'Elia, R. Mock, F. Montori, F. Morandi, L. Rof-fia, S. Rondelli, T. Cinotti and F. Vergari, "An interoperable architecture for mobile smart service over the Internet of energy", 14th IEEE International Symposium and Workshop on World of Wireless, Mobile and Multimedia Networks, (2013).

[7] G. Fabbri, M. Calenne, M. London, C. Boccaletti, A. Marques and F. Mascioli, "Development of an onboard unit for the monitoring and management", International Conference on Electrical Machines, (2008), pp. 2404-2410.

[8] J. Lee, B. Lee, G. Park and Y. Kim, "Web service-based tour-and-charging scheduler framework for renta-car systems employing electric vehicles”, International Journal of Control and Automation, vol. 6, no. 4, (2013), pp. 83-91.

[9] R. Dewri and R. Thurimella, "Exploiting service similarity for privacy in location-based search queries", IEEE Transactions on Parallel and Distributed Systems, vol. 25, no. 2, (2014), pp. 374-383.

[10] J. Lee, H. Kim and G. Park, "Integration of battery charging to tour schedule generation for an EV-based rent-a-car business", Lecture Notes in Computer Science, vol. 7332, (2012), pp. 399-406.

[11] H. Kim, G. Park and J. Lee, "Development of an electric vehicle helper application for emergency rescue", Advanced Technology Letters, vol. 51, (2014) July, pp. 246-249.

[12] M. Poulton and G. Roussos, "Towards smarter metropolitan emergency response", IEEE 24th International Symposium on Personal, Indoor, and Mobile Communications: Mobile and Wireless Networks, (2013), pp. 2576-2580.

[13] R. Loureiro, S. Benmoussa, Y. Touati, R. Merzouki and B. Bouamama, "Integration of fault diagnosis and fault-tolerant control for health monitoring of a class of MIMO intelligent autonomous vehicles", IEEE Transactions on Vehicular Technology, vol. 63, no. 1, (2014), pp. 30-39.

[14] M. Lukasiewycz, S. Steinhorst, F. Sagstetter, W. Chang, P. Waszicki, M. Kauer and S. Chakraborty, "Cyber-physical systems design for electric vehicles", 15th Euromicro Conference on Digital System Design, (2012), pp. 477-484.

[15] S. Bashash and H. Fathy, "Transport-based load modeling and sliding mode control of plug-in electric vehicles for robust renewable power tracking”, IEEE Transactions on Smart Grid, vol. 3, no. 1, (2012), pp. 526-534.

[16] H. Kim and K. Shin, "Scheduling of battery charge, discharge, and rest", IEEE Real-Time Systems Symposium, (2009), pp. 13-22.

[17] H. Kawano, "Applicability of multi-vehicle scheduling problem based on GPS tracking records", International Conference on Geoinformatics, (2010), pp. 1-4.

[18] C. Park, J. Lee, G. Park and J. Hyun, "Development of reservation recommendation algorithms for charging electric vehicles in smart-grid cities", International Journal of Smart Home, vol. 8, no. 1, (2014), pp. 113-122.

[19] J. Lee and G. Park, "Genetic algorithm-based demand response scheme for electric vehicle charging", International Journal of Intelligent Information and Database Systems, vol. 7, no. 6, (2013), pp. 535-549.

[20] M. Vedova, E. Palma and T. Facchinetti, "Electric load as real-time tasks: An application of real-time physical systems", International Wireless communications and mobile computing conference, (2011), pp. 1117-1123.

[21] Y. Ota, J. Taniguchi, T. Nakajima, K. Liyanage, J. Baba and A. Yokoyama, "Autonomous distributed V2G (Vehicle-to-Grid) satisfying scheduled charging”, IEEE Transactions on Smart Grid, vol. 3, no. 1, (2012), pp. 559-564. 
International Journal of Control and Automation Vol.7, No.10 (2014) 